\title{
A Case of Severe Fever With Thrombocytopenia Syndrome Accompanied by Self-Limiting Severe Proteinuria That Inversely Correlated With the Platelet Count
}

\author{
Hiroshi Yamaguchi ${ }^{\mathrm{a}, \mathrm{f}}$, Shuhei Onishi ${ }^{\mathrm{b}}$, Hironobu Shibata ${ }^{\mathrm{c}}$, Kengo Udaka ${ }^{\mathrm{c}}$, Seiji Yoshida ${ }^{\mathrm{d}}$, \\ Takanori Hayakawa ${ }^{\mathrm{e}}$
}

\begin{abstract}
We herein present a case of a 68 -year-old man who was referred to our hospital for a tick bite accompanied by fever and general malaise. He exhibited typical clinical symptoms such as abdominal pain, diarrhea, and vomiting during hospitalization. A laboratory examination showed progressive thrombocytopenia and leukopenia during the early part of hospitalization. A definite diagnosis of severe fever with thrombocytopenia syndrome (SFTS) was made by a reverse transcription polymerase chain reaction (RT-PCR) from his blood sample in the Tokushima Prefectural Public Health Institute. Proteinuria is generally detected in more than half of patients with SFTS. In the present case, the clinical course showed a clear inverse correlation between self-limiting severe proteinuria and the platelet count during hospitalization. Although it is not currently clear how immunological interactions among the virus, platelets, and immunoglobulins affect the glomerulus or cause proteinuria, our results may contribute to elucidating the pathogenic mechanism of SFTS-associated glomerulonephritis. To the best of our knowledge, this case report is the first to show an inverse correlation between proteinuria and the platelet count in a patient with SFTS.
\end{abstract}

Keywords: Severe fever with thrombocytopenia syndrome; Proteinuria; Glomerulonephritis; Tick bite; Thrombocytopenia

Manuscript accepted for publication September 08, 2016

aDepartment of Infectious Diseases, Tokushima Prefectural Central Hospital, Kuramoto, 1-10-3, Tokushima 770-8539, Japan

${ }^{\mathrm{b}}$ The Center for Clinical Education, Tokushima Prefectural Central Hospital, Kuramoto, 1-10-3, Tokushima 770-8539, Japan

'Department of Hematology, Tokushima Prefectural Central Hospital, Kuramoto, 1-10-3, Tokushima 770-8539, Japan

${ }^{\mathrm{d}}$ Department of Respiratory Medicine, Tokushima Prefectural Central Hospital, Kuramoto, 1-10-3, Tokushima 770-8539, Japan

${ }^{\mathrm{e}}$ The Microbiology Laboratory (LSI Medience Corporation), Tokushima Prefectural Central Hospital, Kuramoto, 1-10-3, Tokushima 770-8539, Japan ${ }^{f}$ Corresponding Author: Hiroshi Yamaguchi, Department of Infectious Diseases, Tokushima Prefectural Central Hospital, Kuramoto, 1-10-3, Tokushima 770-8539, Japan. Email: futuyaso007@yahoo.co.jp

doi: http://dx.doi.org/10.14740/jmc2642w

\section{Introduction}

Severe fever with thrombocytopenia syndrome (SFTS) is an emerging infectious zoonosis that was first reported in China in 2011 as a novel bunyavirus infection [1]. The causative agent of this infectious disease is the designated SFTS virus, which belongs to the genus Phlebovirus in the family Bunyaviridae and is carried by ticks (Haemaphysalis longicornis). The first case in Japan was reported in 2013, and as of April 27, 2016, 175 cases have been registered at the National Institute of Infectious Disease (Tokyo, Japan), with a mortality rate of $26.3 \%$ $[2,3]$. Most patients have acute-onset fever and gastrointestinal symptoms such as abdominal pain, diarrhea, vomiting, and anorexia, and typical laboratory findings are thrombocytopenia and leukopenia. Although proteinuria is generally detected in more than half of patients with SFTS, the clinical course of proteinuria has not yet been examined in detail.

\section{Case Report}

We herein present a case of a 68 -year-old man who was referred to our hospital for a tick bite accompanied by acuteonset fever and general malaise. He was still being bitten by the tick on the skin of the left lumbar region at the time of his presentation. He obtained a bamboo shoot from a nearby bamboo grove 4 days before admission. General malaise, such as tired muscles, had developed 2 days before admission. On the day before hospitalization, he visited a nearby clinic with a high fever of $38.2{ }^{\circ} \mathrm{C}$ and nausea in addition to malaise. Gastroenteritis was suspected and, thus, he was prescribed stomach medicine. On the morning of his visit to our hospital, he noticed while he was defecating that a tick had attached to the skin of the lumbar region. The skin around the stab port of the tick was inflamed. Since the tick had strongly attached to the skin, the skin along with the stab port was incised to remove it. Laboratory data revealed decreases in white blood cell (WBC) and platelet (PLT) counts (Table 1).

He had no previous history of major diseases and no blood cell abnormalities were detected in blood tests when he donated blood 1 month prior to admission. Since leukocytopenia and thrombocytopenia in peripheral blood indicated a tickborne infection such as Japanese spotted fever or SFTS, he was immediately hospitalized. At the time of admission, his con- 
Table 1. Laboratory Findings on Admission

\begin{tabular}{|c|c|c|c|}
\hline Urinalysis (third day) & & $\mathrm{CK}$ & $78 \mathrm{U} / \mathrm{L}$ \\
\hline Protein & $2+$ & T-cho & $170 \mathrm{mg} / \mathrm{dL}$ \\
\hline Glucose & - & TG & $52 \mathrm{mg} / \mathrm{dL}$ \\
\hline Ketone bodies & - & $\mathrm{TP}$ & $6.8 \mathrm{~g} / \mathrm{dL}$ \\
\hline Bilirubin & - & Alb & $4.3 \mathrm{~g} / \mathrm{dL}$ \\
\hline Blood cell count & & BUN & $24.7 \mathrm{mg} / \mathrm{dL}$ \\
\hline WBC & $2,500 / \mu \mathrm{L}$ & $\mathrm{Cr}$ & $1.09 \mathrm{mg} / \mathrm{dL}$ \\
\hline $\mathrm{Neu}$ & $78 \%$ & $\mathrm{Na}$ & $139 \mathrm{mEq} / \mathrm{L}$ \\
\hline Eos & $0 \%$ & $\mathrm{~K}$ & $4.5 \mathrm{mEq} / \mathrm{L}$ \\
\hline Baso & $0 \%$ & $\mathrm{Cl}$ & $102 \mathrm{mEq} / \mathrm{L}$ \\
\hline Mono & $11.40 \%$ & Glucose & $104 \mathrm{mg} / \mathrm{dL}$ \\
\hline Lym & $10.60 \%$ & Serological test & \\
\hline $\mathrm{RBC}$ & $469 \times 10^{4} / \mu \mathrm{L}$ & CRP & $0.0 \mathrm{mg} / \mathrm{dL}$ \\
\hline $\mathrm{Hb}$ & $15.3 \mathrm{~g} / \mathrm{dL}$ & HBs Ag & - \\
\hline Plt & $12.9 \times 10^{4} / \mu \mathrm{L}$ & $\mathrm{HCV} \mathrm{Ab}$ & - \\
\hline Blood chemistry & & Hemostatic test (third day) & \\
\hline T-bil & $0.4 \mathrm{mg} / \mathrm{dL}$ & PT & $11.9 \mathrm{~s}$ \\
\hline AST & $19 \mathrm{U} / \mathrm{L}$ & $\mathrm{PT} \%$ & $96 \%$ \\
\hline ALT & $17 \mathrm{U} / \mathrm{L}$ & APTT & $37 \mathrm{~s}$ \\
\hline $\mathrm{LDH}$ & $188 \mathrm{U} / \mathrm{L}$ & Fib & $307 \mathrm{mg} / \mathrm{dL}$ \\
\hline ALP & $231 \mathrm{U} / \mathrm{L}$ & FDP & $5.3 \mu \mathrm{g} / \mathrm{mL}$ \\
\hline AMY & $71 \mathrm{U} / \mathrm{L}$ & & \\
\hline
\end{tabular}

sciousness was clear and he was mentally calm. Although he had a slight headache, other meningeal irritation signs such as a stiff neck and Kernig's sign were negative. The lymph nodes in the neck were not palpated. He did not exhibit erythema, wheal, or petechial rash. On admission, his vital signs were as follows: height, $165 \mathrm{~cm}$; weight, $65 \mathrm{~kg}$; body temperature, $36.1^{\circ} \mathrm{C}$; blood pressure, 129/85 $\mathrm{mm} \mathrm{Hg}$; heart rate, 86 beats/ min with a regular rhythm; respiratory rate, 14 breaths/min; $\mathrm{SpO}_{2}$ 97\% (in room air). The administration of minocycline at a dose of $200 \mathrm{mg} /$ day and levofloxacin at $500 \mathrm{mg} /$ day was initiated immediately in anticipation of rickettsial infections such as Japanese spotted fever, which has frequently appeared in southwest Japan. Laboratory findings through the hospitalization course are shown in Table 2.

Although liver and renal function tests showed no abnormalities at the time of admission, mild liver and renal dysfunctions $(\mathrm{Cr} 1.11 \mathrm{mg} / \mathrm{dL}$, urine protein $2+)$ were detected on the third day of admission. WBC $(1,500 / \mu \mathrm{L})$ and PLT $\left(8.9 \times 10^{4} /\right.$ $\mu \mathrm{L})$ counts on the third day of admission were lower than those at the time of admission. WBC and PLT counts continued to decrease each day. The lowest WBC and PLT counts were noted on the fifth and eighth days after admission, respectively. Se-

Table 2. Clinical Course of Laboratory Examinations

\begin{tabular}{|c|c|c|c|c|c|c|c|c|c|c|c|}
\hline & First & Third & Fourth & Fifth & Sixth & Seventh & Eighth & Ninth & 10th & 12th & 14th \\
\hline $\mathrm{WBC}, / \mu \mathrm{L}$ & 2,500 & 1,500 & 1,300 & 1,100 & 4,000 & 3,300 & 4,700 & 4,900 & 4,900 & 4,600 & 6,300 \\
\hline $\mathrm{Hb}, \mathrm{g} / \mathrm{dL}$ & 15.3 & 15.7 & 15.2 & 14.6 & 16.0 & 15.6 & 16.1 & 15.8 & 15.2 & 15.1 & 15.0 \\
\hline AST, U/L (10 - 35) & 19 & 47 & 86 & 185 & 740 & 572 & 350 & 262 & 197 & 124 & 63 \\
\hline ALT, U/L (5 - 40) & 17 & 32 & 48 & 101 & 538 & 558 & 435 & 405 & 396 & 410 & 301 \\
\hline $\mathrm{Cr}, \mathrm{mg} / \mathrm{dL}(0.5-1.1)$ & 1.09 & 1.11 & 1.08 & 0.89 & 0.82 & 0.78 & 0.73 & 0.69 & 0.80 & 0.70 & 0.78 \\
\hline Ferritin, ng/mL (18.6 - 261) & & & 1,258 & & & & & & & & 550.7 \\
\hline
\end{tabular}




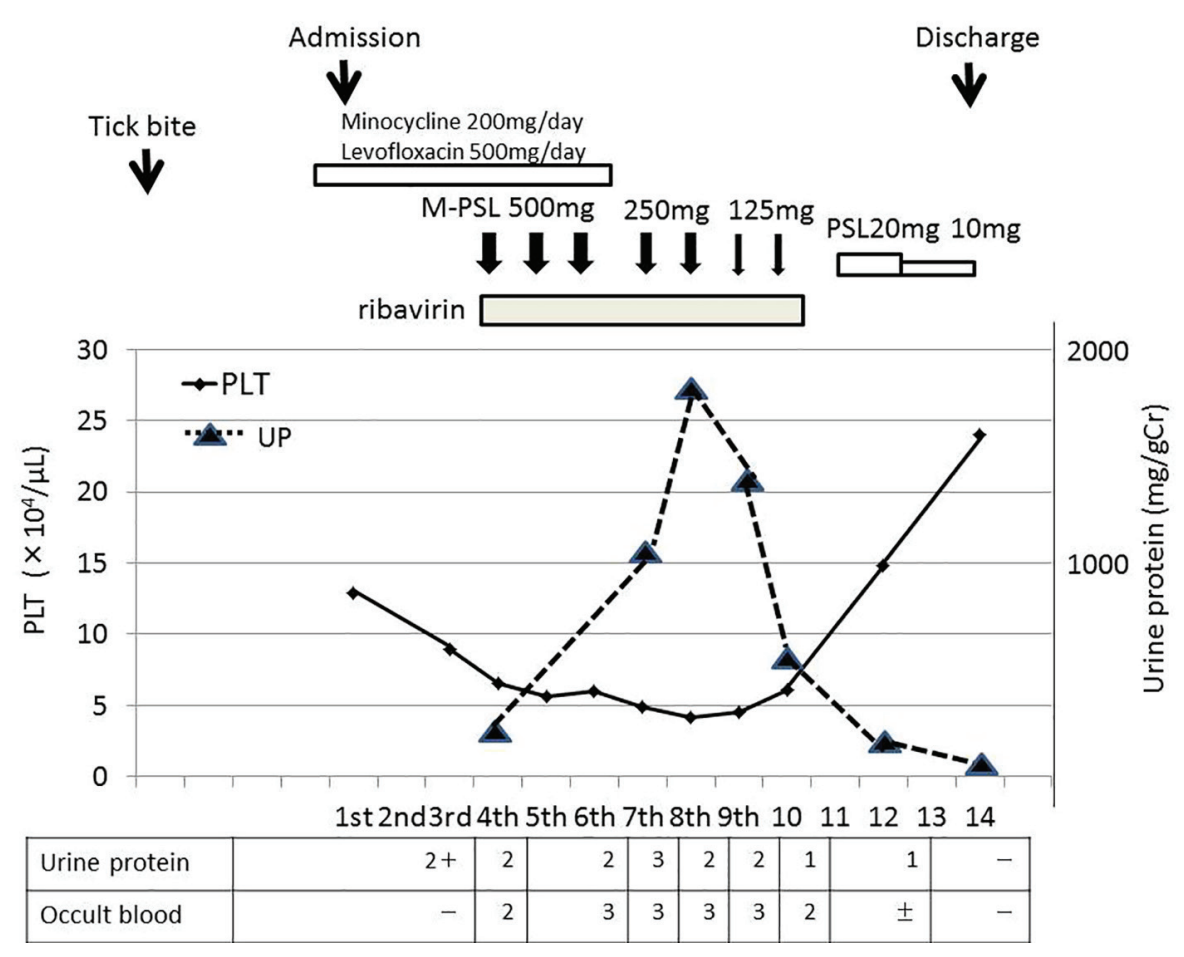

Figure 1. Clinical course of the patient.

rum levels of creatinine kinase (CK) and alanine aminotransferase (ALT) continued to increase after admission, reaching 6,190 and $558 \mathrm{U} / \mathrm{L}$ on the seventh day, respectively. On the third day, we reported this suspected case of SFTS infection to a local public health center because SFTS was designated as a category IV infectious disease under the Infectious Disease Control Law in Japan in 2013 (http://www.nih.go.jp/niid/ja/ iasr.html). On the fourth day, a definite diagnosis of SFTS was made by RT-PCR from his blood sample in the Tokushima Prefectural Public Health Institute. There is currently no standardized treatment for SFTS. Based on previous findings, severe cases of SFTS may quickly proceed to hemophagocytic syndrome (HPS) or multiorgan failure [4, 5]. Immediately after being diagnosed, the patient was started on ribavirin at $600 \mathrm{mg}$ daily for a week and methylprednisolone intravenously at 500 $\mathrm{mg}$ daily for 3 days. Methylprednisolone was then sequentially administered at $250 \mathrm{mg}$ daily for 2 days and $125 \mathrm{mg}$ daily for 2 days, followed by oral prednisolone (Fig. 1).

The administration of prednisolone was finished on the 14th day. On the 10th day, he developed an increasingly frustrated feeling, fell into a state of delirium, and removed the peripheral catheter by himself at night. Therefore, a mental intervention by a psychiatrist was initiated. His mental status gradually improved and he became mentally calm on the 14th day. His urinary protein level gradually increased with decreases in the PLT count, and then gradually decreased with the recovery of the PLT count (Fig. 1). The time course of his urinary protein level and PLT count revealed that they inversely correlated. After the recovery of the PLT count, his urinary protein level improved to below the detection limit on the 14th day.
Although a qualitative immunochemical examination test of urine using reagent test strips showed a positive urinary occult blood test (Fig. 1), the urinary sediments examined under a microscope were within normal range, which is five red blood cells per high power field (RBC/HPF) or less, through the hospitalization course. The time course of variations in $\mathrm{CK}$ showed that when his serum CK level acutely increased on the fourth day after admission, the urinary occult blood test became positive, and when his serum CK level subsequently improved to the normal range on the 14th day, the urinary occult blood test became negative (Table 2 ). Therefore, a positive urinary occult blood test was attributed to a response to myoglobin, which occasionally appears with muscle damage due to viral infection, is excreted by the kidney, and concentrated in the urine. His serum ferritin level was elevated to $1,258 \mathrm{ng} / \mathrm{mL}$ on the fourth day, decreased to $550.7 \mathrm{ng} / \mathrm{mL}$ on the 14 th day, and then normalized to $108 \mathrm{ng} / \mathrm{mL}$ in the follow-up 31 days later. His serum ALT level had returned to within normal range (ALT $20 \mathrm{U} / \mathrm{L}$ ) in the follow-up 31 days later. Although a blood examination associated with nephritis was performed during hospitalization, there was no obvious abnormality (Table 3 ).

Since proteinuria was transient and improved without a specific treatment, renal biopsy was not performed. He was discharged on the 14th day with an improved condition and subsequently followed up as an outpatient at our hospital.

\section{Discussion}

SFTS is generally considered when a patient presents with fever, thrombocytopenia, and leukocytopenia together with a 
Table 3. Blood Examination Associated With Nephritis

\begin{tabular}{llll}
\hline Blood examination & Value & Reference range & \\
\hline $\mathrm{IgG}, \mathrm{mg} / \mathrm{dL}$ & 1,007 & $850-1,850$ & Fourth day \\
$\mathrm{IgA}, \mathrm{mg} / \mathrm{dL}$ & 113 & $90-420$ & Fourth day \\
$\mathrm{IgM}, \mathrm{mg} / \mathrm{dL}$ & 69 & $40-340$ & Fourth day \\
$\mathrm{C} 3, \mathrm{mg} / \mathrm{dL}$ & 114 & $70-130$ & 14 th day \\
$\mathrm{C} 4, \mathrm{mg} / \mathrm{dL}$ & 32 & $15-50$ & 14th day \\
$\mathrm{IC}(\mathrm{C} 1 \mathrm{Qq}), \mu \mathrm{g} / \mathrm{mL}$ & $<1.5$ & $0-3.0$ & Ninth day \\
$\mathrm{CystatinC}, \mathrm{mg} / \mathrm{L}$ & 1.08 & $0.61-1.00$ & Ninth day \\
& 0.92 & & 14 th day \\
ANA & $<\times 40$ & $0-39$ & 12th day \\
$\mathrm{C}-\mathrm{ANCA}, \mathrm{U} / \mathrm{mL}$ & $<1.0$ & $<3.5$ & 12th day \\
$\mathrm{P}-\mathrm{ANCA}, \mathrm{U} / \mathrm{mL}$ & $<1.0$ & $<3.5$ & 12th day \\
RF, IU/mL & Undetectable & $<15$ & 14th day \\
\hline
\end{tabular}

IC: immune complex; ANA: antinuclear antibody; ANCA: anti-neutrophil cytoplasmic antibody; RF: rheumatoid factor.

history of tick exposure in an endemic area. Although all patients with SFTS in Japan have so far occurred in the West, infected areas have gradually been spreading northward. Therefore, people living in infected areas need to take precautions and perform preventive measures against being bitten by an infected tick when gardening or landscaping. A laboratory diagnosis of the SFTS virus is based on a PCR using whole blood or antibody detection in the serum or plasma. In the present case, RT-PCR was performed at a Tokushima health center and a definite diagnosis of SFTS was made. SFTS begins with nonspecific prodromal symptoms, including fever, headache, myalgia, arthralgia, dizziness, and malaise, which persist for approximately 1 week. After prodromal symptoms, patients may develop mucosal hemorrhage, thrombocytopenia, elevated liver enzymes, multiorgan failure, disseminated intravascular coagulopathy (DIC), and central nervous system manifestations such as confusion in severe cases. The present case was suspected of being infected with the SFTS virus at the time of his presentation to our hospital because he had a tick bite in an endemic area and the blood count depression of WBCs and PLTs in blood tests. Patients with SFTS have diverse medical conditions and disease severities. Based on the severity of the SFTS infection, which is generally considered, his medical condition was regarded to be mild to moderate through the hospitalization course. He did not have severe HPS or DIC, and, as a result, did not have prolonged thrombocytopenia or require a PLT transfusion. Therefore, a clear inverse correlation was observed between proteinuria and the PLT count.

There is currently no effective or available antiviral therapy or preventive vaccine against SFTS. Therefore, the main treatment consists of supportive care. Ribavirin, which is used as an antiviral agent to treat hepatitis $\mathrm{C}$, has been reported as an antiviral activity against SFTS in vitro [6]. However, it is not clinically valid when the drug is used as the sole regimen against SFTS infection [7]. On the other hand, combination therapy with ribavirin and other therapeutic agents such as plasma exchange, antibiotics, interferon, and steroids is effec- tive, although evidence for this has only been obtained from a small number of cases [8]. Under the conditions described above, he was treated with combination therapy with ribavirin (600 mg per day for 1 week) and methyl prednisolone pulse therapy (500 $\mathrm{mg}$ per day for 3 days).

A recent study showed that elevated AST, lactate dehydrogenase (LDH) and CK levels, decreased lymphocyte percentages, and an older age correlated with the death of patients with SFTS $[4,5]$. Although thrombocytopenia is a key abnormal finding in SFTS, there have been conflicting findings on the relationship between the PLT count and disease severity [9]. A recent study showed that there was no significant difference in the lowest PLT count during the first week between 32 survival and deceased cases of SFTS in South Korea [10]. Li et al also reported that the PLT counts between both cases were very similar [9]. On the other hand, a study in northeast China showed that the lowest PLT count on admission or throughout hospitalization was associated with the severity of SFTS [11]. In the present case, his PLT count decreased to the lowest value of $41,000 / \mu \mathrm{L}$ on the eighth day, and, thus, he did not need a PLT transfusion. His PLT count then gradually recovered to within normal limits along with improvements in his clinical symptoms such as headache and muscle pain. An assessment of severity by the PLT count was considered difficult in this case because his clinical course was relatively smooth and his condition was non-life-threatening during hospitalization.

The level of SFTS viremia (viral load) has been reported to correlate with disease severity. Deceased patients had significantly higher viral loads than those of survivors when measured on days 7 - 9 after the onset of fever, and viral loads gradually decreased during the recovery phase [9]. Since the microbiology laboratory of our hospital does not meet the facility criteria for dealing with the SFTS virus, we were unable to measure the viral load. Therefore, the relationship between the viral load and disease severity was not clear in this case. Since he was not complicated by severe HPS or DIC, we speculated that he did not have a markedly high viral load. 
Virological and pathological examinations in Japanese patients with SFTS revealed that SFTS virus replicates in blastic cells in the local lymph nodes, but not in the major organs [3, 12]. However, the kidney was not examined in these studies. In an infectious mouse model of SFTS in C57/BL6, viral RNA and histopathological changes were identified in the spleen, liver, and kidney, and viral replication was only detected in the spleen among those organs [13]. Although the spleen is the main target organ of the SFTS virus in a mouse model, it differs from the above-described autopsy findings in humans. A pathological examination of the kidney of the mouse model revealed glomerular hypercellularity, mesangial thickening, and the congestion of Bowman's space. However, the infiltration of inflammatory cells was absent in the kidney. These pathological changes were maximized on the 14th day after infection, and recovered to nearly normal levels on the 28th day. The pathological changes observed in the kidney at the later stage of infection appear to be indicative of acute glomerular nephritis with self-limiting outcomes and are consistent with the clinical course of proteinuria in this case.

In the laboratory testing of patients with SFTS in China, proteinuria and hematuria were detected in $84 \%$ and $59 \%$ of patients, respectively [1]. Glomerulonephritis associated with several viral infections such as hepatitis B and $\mathrm{C}$ and human immunodeficiency virus (HIV) is well known. In terms of its mechanism, glomerular injury may be due to the formation of circulating immune complexes involving viral antigens and antibodies, the formation of in situ antigen-antibody reactions, and autoimmune reactions to glomerular structures induced by the virus $[14,15]$. However, the mechanism of transient proteinuria associated with SFTS viral infections has not yet been investigated.

Dengue virus infection, the most prevalent mosquitoborne viral disease in tropical and subtropical countries worldwide that is typically accompanied by thrombocytopenia and leukopenia, has been associated with a number of renal disorders from acute renal failure to asymptomatic proteinuria or hematuria. Horvath et al reported that proteinuria was detected in as many as $74 \%$ of patients with severe dengue infection $[16,17]$. Although severe proteinuria such as nephrotic-range proteinuria is a rare complication in patients with dengue virus infection, there have been a few case reports that are similar to our SFTS case. Hebbal et al described an 8-year-old boy with severe dengue complicated by nephrotic-range proteinuria. Proteinuria and the PLT count inversely correlated, followed by spontaneous resolution with full recovery [14]. Vasanwala et al also reported two Dengue hemorrhagic fever patients with nephrotic-range proteinuria $(8.0$ - $9.0 \mathrm{~g} /$ day $)$ [18]. These patients did not have hematuria or elevated serum creatinine concentrations, and transient nephrotic-range proteinuria improved without specific treatments $[14,18,19]$. Various types of dengue-associated glomerulonephritis have been reported in humans and mouse models of dengue infection. In most cases, dengue infection and its associated renal disorders are selflimiting. Possible mechanisms causing self-limiting proteinuria have been investigated pathologically and immunologically. Previous studies reported that mesangial proliferation and immune complex deposition are dominant histological features $[17,18]$. Self-limiting proteinuria associated with
SFTS virus infection has not yet been examined. Based on the above-described mechanism, the pathogenic mechanism of SFTS-associated glomerulonephritis may also be autoimmune.

A recent study showed that the Dengue virus binds to human PLTs and replicates its viral RNA utilizing their translation mechanism. Since the Dengue virus has positive-sense single-stranded RNA $((+)$ ssRNA), it is speculated that other positive-sense ssRNA viruses may be similarly propagated within the anucleate cytoplasm of PLTs $[20,21]$. On the other hand, the SFTS virus, which belongs to family Bunyaviridae, is a negative-sense ssRNA virus ((-)ssRNA virus), and, thus, it currently remains unclear whether the SFTS virus replicates within the cytoplasm of PLTs. In the SFTS mouse model, the SFTS virus was shown to adhere to PLTs and the adhered PLTs enhanced phagocytosis by macrophages. In addition, the viral RNA of the SFTS virus was detected in the kidney. Although these findings obtained from the mouse model are circumstantial evidence, SFTS-associated glomerulonephritis may be caused by immunological mechanisms in glomerular cells after the SFTS virus has attached to PLTs and been incorporated into macrophages.

\section{Conclusion}

We herein present a case of a 68 -year-old man with SFTS accompanied by self-limiting severe proteinuria. Although it is not currently clear how immunological interactions among the virus, PLTs, and immunoglobulins affect the glomerulus or cause proteinuria, our results may contribute to elucidating the pathogenic mechanism of SFTS-associated glomerulonephritis. To the best of our knowledge, this is the first case report showing an inverse correlation between proteinuria and the PLT count in a patient with SFTS.

\section{Consent}

Written informed consent was obtained from the patient for the publication of this case report.

\section{Conflicts of Interest}

The authors declare no conflicts of interest.

\section{References}

1. Yu XJ, Liang MF, Zhang SY, Liu Y, Li JD, Sun YL, Zhang L, et al. Fever with thrombocytopenia associated with a novel bunyavirus in China. N Engl J Med. 2011;364(16):1523-1532.

2. Infectious Agents Surveillance Report; http://www.nih. go.jp/niid/en/component/content/article/865-iasr/6339tpc433.html.

3. Takahashi T, Maeda K, Suzuki T, Ishido A, Shigeoka T, Tominaga T, Kamei T, et al. The first identification and 
retrospective study of Severe Fever with Thrombocytopenia Syndrome in Japan. J Infect Dis. 2014;209(6):816827.

4. Kitao A, Ieki R, Takatsu H, Tachibana Y, Nagae M, Hino T, Nakaji H, et al. Severe fever with thrombocytopenia syndrome presenting as hemophagocytic syndrome: two case reports. Springerplus. 2016;5:361.

5. Sun Y, Jin C, Zhan F, Wang X, Liang M, Zhang Q, Ding $\mathrm{S}$, et al. Host cytokine storm is associated with disease severity of severe fever with thrombocytopenia syndrome. J Infect Dis. 2012;206(7):1085-1094.

6. Shimojima M, Fukushi S, Tani H, Yoshikawa T, Fukuma A, Taniguchi S, Suda Y, et al. Effects of ribavirin on severe fever with thrombocytopenia syndrome virus in vitro. Jpn J Infect Dis. 2014;67(6):423-427.

7. Liu W, Lu QB, Cui N, Li H, Wang LY, Liu K, Yang ZD, et al. Case-fatality ratio and effectiveness of ribavirin therapy among hospitalized patients in china who had severe fever with thrombocytopenia syndrome. Clin Infect Dis. 2013;57(9):1292-1299.

8. Shimojima M, Fukushi S, Tani H, Taniguchi S, Fukuma A, Saijo M. Combination effects of ribavirin and interferons on severe fever with thrombocytopenia syndrome virus infection. Virol J. 2015;12:181.

9. Li J, Han Y, Xing Y, Li S, Kong L, Zhang Y, Zhang L, et al. Concurrent measurement of dynamic changes in viral load, serum enzymes, $\mathrm{T}$ cell subsets, and cytokines in patients with severe fever with thrombocytopenia syndrome. PLoS One. 2014;9(3):e91679.

10. Shin J, Kwon D, Youn SK, Park JH. Characteristics and Factors Associated with Death among Patients Hospitalized for Severe Fever with Thrombocytopenia Syndrome, South Korea, 2013. Emerg Infect Dis. 2015;21(10):17041710 .

11. Deng B, Zhou B, Zhang S, Zhu Y, Han L, Geng Y, Jin Z, et al. Clinical features and factors associated with severity and fatality among patients with severe fever with throm- bocytopenia syndrome Bunyavirus infection in Northeast China. PLoS One. 2013;8(11):e80802.

12. Hiraki T, Yoshimitsu M, Suzuki T, Goto Y, Higashi M, Yokoyama S, Tabuchi T, et al. Two autopsy cases of severe fever with thrombocytopenia syndrome (SFTS) in Japan: a pathognomonic histological feature and unique complication of SFTS. Pathol Int. 2014;64(11):569-575.

13. Jin C, Liang M, Ning J, Gu W, Jiang H, Wu W, Zhang F, et al. Pathogenesis of emerging severe fever with thrombocytopenia syndrome virus in C57/BL6 mouse model. Proc Natl Acad Sci U S A. 2012;109(25):10053-10058.

14. Hebbal P, Darwich Y, Fong J, Hagmann SH, Purswani MU. Nephrotic-range proteinuria in an eight-year-old traveler with severe dengue: Case report and review of the literature. Travel Med Infect Dis. 2016;14(1):45-48.

15. Lohr JW, Batuman V. Glomerulonephritis Associated with Nonstreptococcal Infection. emedicine.medscape. com/article/240229-overview.

16. Horvath R, McBride WJH, Hanna J. Clinical features of hospitalized patients during Dengue-3 epidemic in far north Queensland 1997-1999. Dengue Bulletin. 1999;23:24-29.

17. Lizarraga KJ, Nayer A. Dengue-associated kidney disease. J Nephropathol. 2014;3(2):57-62.

18. Vasanwala FF, Puvanendran R, Ng JM, Suhail SM. Two cases of self-limiting nephropathies secondary to dengue haemorrhagic fever. Singapore Med J. 2009;50(7):e253255.

19. Hutspardol S, Prommalikit O, Upiya N, Chataroopwijit J, Khemakanok K, Assadamongkol K. Heavy proteinuria following dengue hemorrhagic fever. Southeast Asian J Trop Med Public Health. 2011;42(3):579-582.

20. Simon AY, Sutherland MR, Pryzdial EL. Dengue virus binding and replication by platelets. Blood. 2015;126(3):378-385.

21. Rondina MT, Weyrich AS. Dengue virus pirates human platelets. Blood. 2015;126(3):286-287. 